\title{
Análisis para la Implementación del Sistema de Gestión de Calidad y del Sistema de Gestión Ambiental para el Laboratorio de Análisis Instrumental de la Escuela Politécnica Nacional
}

\author{
Delgado, Mónica Susana ${ }^{1,2,3}$; Cabrera, Marcelo ${ }^{1,2}$; Pérez, Gabriela ${ }^{1}$ \\ ${ }^{1}$ Escuela Politécnica Nacional, Quito, Ecuador \\ ${ }^{2}$ Universidad Internacional SEK, Facultad de Ciencias Naturales y Ambientales, Quito, Ecuador \\ ${ }^{3}$ Universidad de Valencia, Valencia, España
}

\begin{abstract}
Resumen: El Laboratorio de Análisis Instrumental ubicado en el 5to piso del Edificio No. 17 de la Escuela Politécnica Nacional brinda servicios de análisis de gases de pozo, biogás, biocidas, solventes, vidrios laminados y templados, entre otros; no obstante, no cuenta con un sistema integrado de Gestión que incluya la ISO 9001:2015 ni ISO 14001:2015, los cuales deberían ser una base fundamental a fin de permitir una dirección basada en la optimización de procesos con una disminución de los impactos ambientales que puede generar cualquier empresa. Con estos antecedentes, el presente trabajo tiene por objetivo desarrollar un mecanismo para implementación de un SIG y que vaya acorde a la normativa ISO 17025:2015 mediante una búsqueda sistemática de bibliografía y como parte de la acreditación del mismo. Los resultados obtenidos muestran que la técnica de integración total, propuesta por Block y Marash (2000) y la técnica de alineamiento propuesta por Ferguson et al (2002), son adecuadas para este tipo de laboratorio, y replicables para laboratorios similares.
\end{abstract}

Palabras clave: Calidad, medio ambiente, laboratorio de análisis instrumental, sistemas integrados de gestión.

\section{Analysis for the Implementation of the Quality Management System and the Environmental Management System for the Instrumental Analysis Laboratory of the National Polytechnic School}

\begin{abstract}
The Instrumental Analysis Laboratory located on the 5th floor of Building No. 17 of the National Polytechnic School provides services for the analysis of well gases, biogas, biocides, solvents, laminated and tempered glass, among others; however, it does not have an integrated management system that includes ISO 9001: 2015 nor ISO 14001: 2015, which should be a fundamental basis in order to allow management based on process optimization with a reduction in environmental impacts what any company can generate With this background, this work aims to develop a mechanism for implementing a GIS and that is consistent with ISO 17025: 2015 by a systematic search of literature and as part of the accreditation of it. The results obtained show that the technique of total integration, proposed by Block and Marash (2000) and the alignment technique proposed by Ferguson et al (2002), are suitable for this type of laboratory, and replicable for similar laboratories.
\end{abstract}

Keywords: Quality, environment, instrumental analysis laboratory, integrated management systems.

\section{CASO DE ESTUDIO}

El Laboratorio de Análisis Instrumental (LAI) ubicado en el 5to piso del Edificio número 17 de la Escuela Politécnica Nacional brinda servicios de análisis de composición de gases de pozo, biogás, biocidas, solventes, índices de protección UV de vidrios laminados y templados, entre otros; no obstante, no cuenta con un sistema integrado de gestión (SIG) a pesar de que está en proceso de la acreditación de laboratorio bajo la norma ISO 17025.
Para ello, el LAI cuenta con 2 cromatógrafos de gases marcas Perkin Elmer y Agilent, un equipo de espectrofotometría de Ultravioleta marca Thermo Científic, tal y como se puede evidenciar en las Figuras 1, 2, 3.

SIG permitiría una dirección basada en la optimización de procesos con una disminución de los impactos ambientales generados por el laboratorio y adicional a ello precautelar la salud de todo el personal del Laboratorio. 


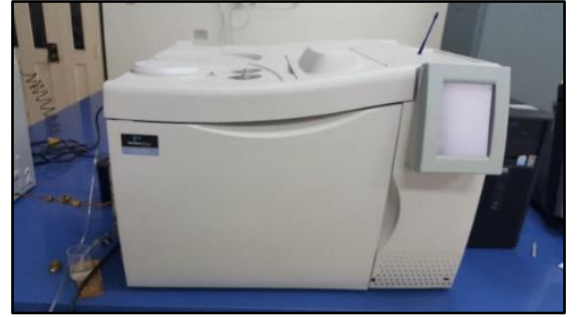

Figura 1.Cromatógrafo de Gases del LAI marca Perkin-Elmer

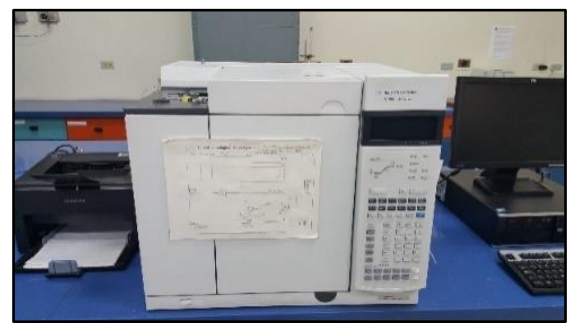

Figura 2. Cromatógrafo de Gases del LAI marca Agilent Technologies

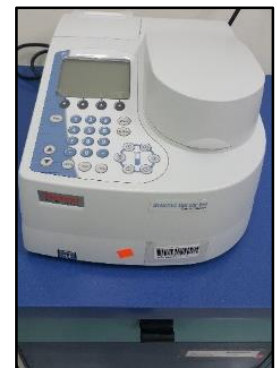

Figura 3. Espectrofotómetro Ultravioleta marca Thermo Scientific

La misión del Laboratorio de Análisis Instrumental de la Escuela Politécnica Nacional es el manejar una política de calidad que permita brindar un servicio a sus clientes enmarcados en la calidad en todos los ensayos que se realizan, alineados con los estándares y normas respectivas (Escuela Politécnica Nacional, 2017).

\section{METODOLOGÍA}

\subsection{Revisión Bibliográfica. Preámbulo}

La cuenca hidrográfica del Cañar $\left(2246 \mathrm{~km}^{2}\right)$ se ubica en la zona centro occidental del Ecuador. La cuenca alta tiene una topografía fuertemente accidentada conformada, por la cordillera occidental de los Andes, y descendiendo abruptamente la elevación del terreno hasta llegar a una topografía plana en el punto de cierre de la cuenca a $19 \mathrm{msnm}$. (Figura 1).

En la actualidad, el LAI está en proceso de acreditación mediante la norma NTE INEN-ISO/IEC 17025:2005, Este aval es emitido por el Servicio de Acreditación Ecuatoriano en cumplimiento con los requisitos establecidos en la Norma NTE - INEN ISO/IEC 17025:2006 "Requisitos generales para la competencia de los laboratorios de ensayo y calibración" equivalente a la Norma ISO/IEC 17025: 2005 y con los criterios y procedimientos de acreditación del SAE (Servicio de Acreditación Ecuatoriano). Se está desarrollando el Manual de Calidad con los respectivos procedimientos generales y procedimientos específicos, los cuales se debe cumplir en dicha normativa (INEN, 2005).
Por lo cual se realiza una revisión sistemática y bibliográfica, que demuestra que el plantear un Sistema Integrado de Gestión, a partir de la estructura ISO, es relativamente sencillo y exitoso si se considera que:

"El éxito de un proceso de integración de sistemas de gestión viene condicionado en gran medida por la implicación y apoyo mostrados por la Alta Dirección, tanto en el momento de toma de decisiones como durante el desarrollo e implantación del mismo" (Abad, 2011, pp. 85-91).

Ahora bien, se puede volver un sistema integrado de gestión inmanejable cuando los procesos que involucran el desarrollo de las actividades se vuelven complicados (Fox, Chionglo, y Barbuceanu, 2013). Claro ejemplo es el caso de estudio, al ser un Laboratorio que pertenece al Sector Público, éste no posee independencia para poder llevar a cabo todo lo relacionado con la implementación de un SIG, ya que depende de otras áreas como son: Contabilidad, Facturación, Talento Humano, Seguridad y Salud Ocupacional, las cuales no necesariamente se manejan con un Sistema Integrado de Gestión propio.

Dada la dificultad planteada, se evalúan 116 artículos científicos relacionados, en bases de datos como science direct, scopus, PubMed, Lilacs, SciELO, Google Scholar, con 5 años de antigüedad y en su mayoría open sources, a fin de encontrar la estrategia más adecuada para la implementación.

\subsection{Estrategia de implementación:}

\section{Propuesta de Block y Marash (2000)}

Esta propuesta está enfocada a la integración de SIG a partir de los estándares ISO 9000:2015 e ISO 14001:2015, mediante 2 metodologías:

\section{a. Integración parcial}

Considerando para cada sistema de trabajo un manual, que contiene documentos compartidos y procedimientos entre el resto de sistemas, el sistema de gestión ambiental utiliza los procedimientos del sistema de gestión de calidad que se pueden aplicar al medioambiente, mediante las modificaciones necesarias.

\section{b. Integración total}

En este caso los sistemas pierden identidad ya que cualquier procedimiento se modifica a fin de facilitar el trabajo de aplicación, y este es plasmado en un documento.

\section{Propuesta de Ferguson et al. (2002)}

Se consideran tres posibles niveles:

\section{a. Alineamiento}

Los sistemas están bajo la jurisdicción de un departamento o unidad y cada una de ellas sigue sus propios objetivos y el SIG es solo documental. 


\section{b. Combinación}

Existe la separación por departamentos o unidades, pero algunos procedimientos son comunes.

c. Integración

Existe el sistema completamente integrado en todas las áreas: calidad, ambiente y seguridad industrial.

\section{RESULTADOS Y DISCUSIÓN}

\subsection{Diagrama de proceso del LAI.}

En la Figura 4 se muestra el proceso LAI como parte de un Departamento que es el de Ingeniería Química, y además es parte de un Sistema Global que es el de la EPN.

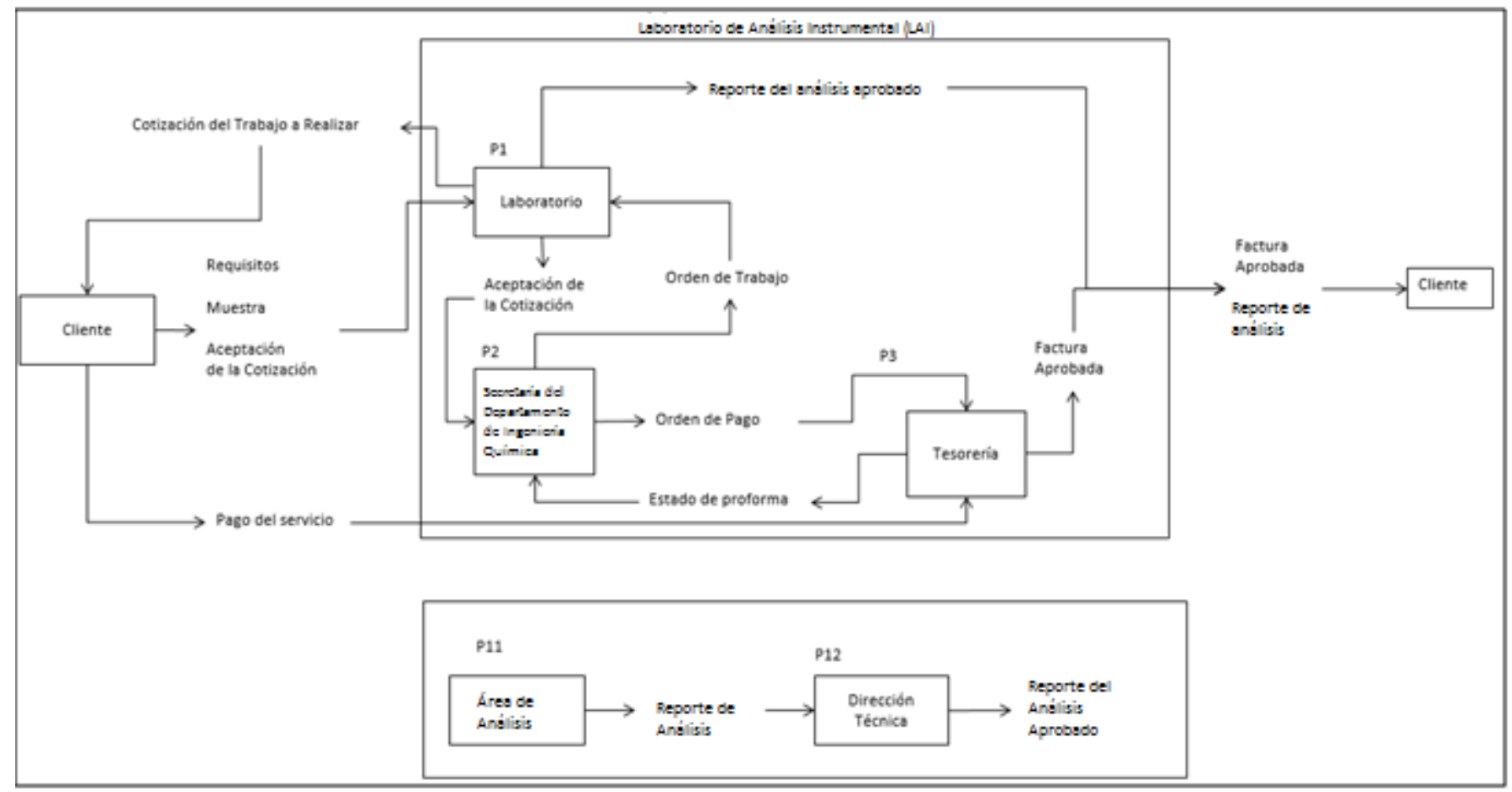

Figura 4. Diagrama de proceso del Laboratorio de Análisis Instrumental de la Escuela Politécnica Nacional

La Escuela Politécnica Nacional (LAI) realiza ensayos de diversos tipos para las industrias, los cuales comprenden desde caracterizaciones físico-químicas de los productos hasta determinación de sus composiciones mediante técnicas cromatográficas.

Para lo cual, el cliente realiza una solicitud de análisis vía email y el laboratorio le envía la cotización correspondiente, una vez que la cotización es aceptada por el cliente, éste lo notifica y entrega la muestra al LAI, el cual llena un registro de ingreso y notifica la aceptación de la cotización a la secretaria del Departamento de Ingeniería Química (DIQ), ésta genera una orden de pago, para su posterior envió al Departamento de Tesorería.

La Tesorería se encarga de recibir el pago del cliente para acceder al servicio. Se le emite la factura y cambia el estado de la proforma en el Sistema Integrado de Información a "Facturada", dicho estado es recibido por la secretaria del DIQ y envía una orden de trabajo al LAI, el mismo que procede a realizar el análisis pertinente y entrega el informe aprobado al cliente.

\subsection{Resultado de la estrategia aplicada:}

Se tomó de referencia la propuesta de Block y Marash (2000) como paso 1 , porque permite la integración de 2 normativas, que es la de calidad y la de gestión ambiental, muy ligadas al sistema que está en proceso de implementación que es la ISO 17025:2005.

En virtud de ello, se ha decidido realizar una integración total, ya que se desea tener un solo manual en el que consten los requisitos combinados de gestión ambiental y de calidad (Abad, 2017). Se puede observar el resultado de la integración de los sistemas en la Tabla 1: 
Tabla 1. Requisitos de las normas ISO

\begin{tabular}{|c|c|c|c|}
\hline Elemento & Tema & 9001:2015 & 14001:2015 \\
\hline 4 & \multicolumn{3}{|c|}{ CONTEXTO DE LA ORGANIZACIÓN } \\
\hline 4.1 & $\begin{array}{l}\text { Contexto General } \\
\text { del Laboratorio. }\end{array}$ & $\begin{array}{l}\text { Generalidades externas e internas que afectan a la } \\
\text { gestión de calidad y seguimiento }\end{array}$ & $\begin{array}{l}\text { Generalidades externas e internas que afectan a la } \\
\text { gestión ambiental }\end{array}$ \\
\hline 4.3 & $\begin{array}{l}\text { Determinación de } \\
\text { alcance del } \\
\text { SGC/SGA }\end{array}$ & Establecer el alcance considerando $4.1,4.2$ & Establecer el alcance considerando $4.1,4.2$ \\
\hline 5 & \multicolumn{3}{|c|}{ LIDERAZGO } \\
\hline 5.2 & $\begin{array}{l}\text { Política General y } \\
\text { ambiental }\end{array}$ & $\begin{array}{c}\text { Rectorado/ o su delegado debe establecer la política de } \\
\text { calidad que regirá en toda la universidad, así como el } \\
\text { laboratorio se encargará de implementarla y mantenerla } \\
\text { en armonía con los objetivos de calidad, compromisos y } \\
\text { mejoras continuas. }\end{array}$ & $\begin{array}{l}\text { Rectorado/ o su delegado debe establecer la política } \\
\text { ambiental que regirá en toda la universidad y la } \\
\text { definición de su SGA que incluya los compromisos } \\
\text { para la protección del medio ambiente. }\end{array}$ \\
\hline 6 & \multicolumn{3}{|c|}{ PLANIFICACIÓN } \\
\hline 6.1.1 & $\begin{array}{l}\text { Planificación } \\
\text { general, FODA }\end{array}$ & $\begin{array}{l}\text { Considerar el } 4.1,4.2 \text {, en la revisión de riesgos y } \\
\text { oportunidades a fin de generar una mejora continua. }\end{array}$ & $\begin{array}{c}\text { Considerar el 4.1, 4.2, riesgos y oportunidades } \\
\text { relacionados con la prevención de riesgos ambientales y } \\
\text { en relación con los aspectos. }\end{array}$ \\
\hline 7 & \multicolumn{3}{|r|}{ 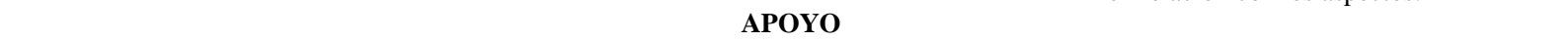 } \\
\hline 7.1.1 & $\begin{array}{l}\text { Apoyo. Recursos. } \\
\text { Generalidades }\end{array}$ & $\begin{array}{c}\text { Se considera las limitaciones de los recursos existentes, y } \\
\text { se amplía el grado de acción con proveedores externos } \\
\text { en caso de ser necesario. }\end{array}$ & $\begin{array}{l}\text { Se establecen los recursos para el cumplimiento del } \\
\text { SGA. }\end{array}$ \\
\hline 7.2 & Competencia & $\begin{array}{c}\text { Se busca la competencia, de tal manera que abarque } \\
\text { educación, formación o experiencia del personal de } \\
\text { laboratorio en la gestión de Calidad. }\end{array}$ & $\begin{array}{l}\text { Se busca la competencia, de tal manera que abarque } \\
\text { educación, formación o experiencia del personal que } \\
\text { manejará la gestión del SGA para el laboratorio. }\end{array}$ \\
\hline 7.4.1 & Comunicación & $\begin{array}{c}\text { Determinar el mecanismo de comunicaciones internas y } \\
\text { externas que respondan a las preguntas que, cuando, a } \\
\text { quien, como y quien comunica }\end{array}$ & $\begin{array}{c}\text { Determinar el mecanismo de comunicaciones internas } \\
\text { y externas pertinentes al SGA que respondan a las } \\
\text { preguntas que, cuando, a quien, como y quien } \\
\text { comunica. }\end{array}$ \\
\hline 7.5.1 & $\begin{array}{l}\text { Información } \\
\text { documentada }\end{array}$ & $\begin{array}{l}\text { EL SGC debe incluir la documentación requerida por la } \\
\text { ISO 9001:2015 y su eficacia }\end{array}$ & $\begin{array}{l}\text { EL SGA debe incluir la documentación requerida por la } \\
\text { ISO 14001:2015 y su eficacia }\end{array}$ \\
\hline 8 & \multicolumn{3}{|c|}{ OPERACIÓN } \\
\hline 8.1 & $\begin{array}{l}\text { Operación: } \\
\text { Planificación y } \\
\text { Control operacional }\end{array}$ & $\begin{array}{l}\text { Se establecen y planifican los requisitos de calidadpara } \\
\text { los productos y servicios }\end{array}$ & $\begin{array}{c}\text { Implementar acciones determinadas en los apartados } \\
6.1 \text { y } 6.2 \text { de la norma. Se controlará los cambios, y el } \\
\text { ciclo de vida de los productos y servicios del } \\
\text { laboratorio, así como la comunicación a todos los } \\
\text { involucrados }\end{array}$ \\
\hline 9 & \multicolumn{3}{|c|}{ EVALUACIÓN DEL DESEMPEÑO } \\
\hline 9.1.1 & Generalidades & $\begin{array}{l}\text { Determinar donde se evaluará y donde se realizará el } \\
\text { seguimiento de calidad en los procesos del laboratorio. }\end{array}$ & $\begin{array}{l}\text { Realizar seguimiento, medir, analizar y evaluar el } \\
\text { desempeño y eficacia del SGA, Se debe comunicar } \\
\text { interna y externamente según corresponda la } \\
\text { información referente al desempeño ambiental y } \\
\text { mantener la información documentada apropiada }\end{array}$ \\
\hline 9.2 .1 & Auditoría Interna & $\begin{array}{l}\text { Realiza auditorías internas que cubren todos los aspectos } \\
\text { de calidad a fin de mantener la eficacia y eficiencia del } \\
\text { sistema. }\end{array}$ & $\begin{array}{c}\text { Realiza auditorías internas que cubren todos los } \\
\text { aspectos de SGA a fin de mantener la eficacia y } \\
\text { eficiencia del sistema. }\end{array}$ \\
\hline 9.2 .2 & Auditoría Interna & $\begin{array}{l}\text { Las auditorías incluirán la frecuencia, métodos, } \\
\text { responsabilidades, requisitos de planificación y } \\
\text { elaboración de informes internos. }\end{array}$ & $\begin{array}{l}\text { Para esto se definirán los criterios de la auditoría, la } \\
\text { objetividad, la comunicación y documentación. }\end{array}$ \\
\hline 10 & \multicolumn{3}{|c|}{ MEJORA } \\
\hline 10.2.1 & $\begin{array}{l}\text { No conformidad y } \\
\text { acción correctiva. }\end{array}$ & $\begin{array}{c}\text { Se dará pronta respuesta a las quejas, y se las procesará } \\
\text { de acuerdo a los alcances del laboratorio. }\end{array}$ & $\begin{array}{l}\text { Se mitigarán impactos, y se corregirá acciones } \\
\text { relacionadas al complimiento del SGA. }\end{array}$ \\
\hline 10.3 & Mejora Continua & $\begin{array}{c}\text { Mejorar continuamente la conveniencia, adecuación y } \\
\text { eficacia en la calidad y considerar los resultados, } \\
\text { necesidades y oportunidades }\end{array}$ & $\begin{array}{l}\text { Mejorar continuamente la conveniencia, adecuación y } \\
\text { eficacia del SGA para mejorar el desempeño ambiental. }\end{array}$ \\
\hline
\end{tabular}

\section{Propuesta de Ferguson et al. (2002)}

Como paso 2, Dada la interrelación de varios departamentos y unidades externas al Laboratorio tal y como se puede ver en la Figura 4, se decidió complementar con la propuesta de Ferguson (2002), debido a que sería complicado solicitar la implementación de un SIG a los departamentos aledaños.

Según González (2011), es importante considerar el ciclo Deming para la mejora continua (pp. 70-71), es por ello que a continuación se presenta el análisis desarrollado para el LAI:

\section{Objetivo General}

Manejar una política de calidad que permita brindar un servicio que cumpla con los requisitos y expectativas del cliente en todos los ensayos que se realizan, alineados con los estándares y normas respectivos.

\section{Objetivos Específicos}

- Cumplir con los tiempos establecidos en los procesos de análisis y en la entrega del reporte aprobado al cliente.

- Realizar los ensayos de laboratorio basados en las normas nacionales e internacionales vigentes. 


\section{Planificar}

Realizar los ensayos solicitados por el cliente mediante el cumplimiento con las normas respectivas y la entrega de los resultados del análisis dentro del tiempo establecido en la proforma aceptada por el cliente.

\section{Hacer}

1. Emplear normas vigentes en la realización de los ensayos.

2. Capacitar al personal en las técnicas de análisis.

3. Mejorar la comunicación entre los Departamentos.

4. Facilitar el uso de sistemas de tecnologías de la información a todo el personal del Laboratorio.

\section{Verificar}

1. Llevar un registro la vigencia de las normas cada 6 meses.

- Ejecutor: Especialista en técnicas de Análisis Químico.

- Recursos: El Departamento Financiero entregará los recursos económicos en caso de requerir adquirir normas vigentes, previa autorización de la Dirección Administrativa.

- Responsable: Jefe del Departamento de Ingeniería Química.

2. Emplear un registro de capacitación de los trabajadores por ensayo anualmente.

- Ejecutor: Especialista en técnicas de Análisis Químico.

- Recursos: El Departamento Financiero entregará los recursos económicos en caso de requerir adquirir normas vigentes, previa autorización de la Dirección Administrativa.

- Responsable: Director Técnico del Laboratorio.

3. Formalizar la comunicación mediante el empleo de correos electrónicos y no emplear únicamente la comunicación verbal.

- Ejecutor: Especialista en técnicas de Análisis Químico.

- Recursos: El Departamento de Gestión de Procesos de la Información se encargará de asegurar un correcto funcionamiento de las redes inalámbricas.

- Responsable: Especialista en técnicas de Análisis Químico.

4. Realizar una solicitud que permita la instalación de programas informáticos que faciliten la visualización en el cambio del estado de las proformas.

- Ejecutor: Secretaria del Departamento de Ingeniería Química.

- Recursos: El Departamento de Gestión de Procesos de la Información otorgará los programas informáticos necesarios.

- Responsable: Jefe del Departamento de Ingeniería Química.

\section{Actuar}

Implementar nuevas técnicas de análisis para ensayos que actualmente no se realizan en el país mediante la adquisición de nuevos equipos a fin de desarrollar ensayos con tecnología de punta y de esta forma incrementar la investigación y mejorar la economía del país.

\section{CONCLUSIONES Y RECOMENDACIONES}

Para aplicar un Sistema Integrado de Gestión al Laboratorio de Análisis Instrumental de la Escuela Politécnica Nacional, se ha decidido utilizar 2 metodologías: una integración total, propuesta por Block y Marash (2000) debido a que se desea tener un solo manual en el que consten los requisitos combinados de gestión ambiental y de calidad.

La otra metodología utilizada es la técnica de alineamiento propuesta de Ferguson et al., ya que es complicado solicitar la implementación de un SIG a los departamentos aledaños.

Los principales procesos que presentan riesgos para el SGC están dados en las operaciones de la realización del ensayo y análisis de resultados, los cuales pueden ser controlados especialmente con capacitación.

Los principales procesos que presentan riesgos para el SGA están dados en las operaciones de la preparación de la muestra y realización del ensayo, los cuales pueden ser controlados especialmente con una revisión periódica del estado de las válvulas de los tanques de alta presión y capacitación.

La mejora continua es importante mantenerla con la finalidad de que se puedan optimizar cada vez más los procesos, recursos, insumos, etc., de un laboratorio.

Se recomienda prestar especial atención al sistema de auditorías internas por lo que esto permitiría una adecuada implementación del SIG propuesto.

\section{REFERENCIAS}

Abad, J. (2011). Implicaciones de la integración de los sistemas de gestión de Calidad, medio ambiente y seguridad y salud laboral basados en estándares internacionales (Tesis Doctoral). UniversitatPolitècnica de Catalunya, España

Abad, P. y Rodríguez M. P. (2017). La Integración de Sistemas de Gestión: un Concepto Indefinido. Recuperado de: https://www.prevencionintegral.com/canal-orp/papers/orp-

2006/integracion-sistemas-gestion-un-concepto-indefinido. (Octubre 2017).

Block, M. R. y Marash, I. R. (2000). Integración de ISO 14001 en un Sistema de Gestión de la Calidad. Madrid: AENOR.

Escuela Politécnica Nacional. (2017). Laboratorio de Análisis Instrumental. Recuperado de: http://www.epn.edu.ec/laboratorio-de-analisisinstrumental/. (Octubre 2017).

Ferguson, M., García, M. y Bornay, M. (2002). "Modelos de implantación de los sistemas integrados de gestión de la calidad, el medio ambiente y la seguridad", Investigaciones Europeas de Dirección y Economía de la Empresa, Vol. 8, No. 1, pp. 97-118.

Fox, M., Chionglo, J. y Barbuceanu M. (2013). "The Integrated Supply Chain Management System", Recuperado de:http://eil.utoronto.ca/wpcontent/uploads/public/papers/iscm-intro.pdf. (Octubre, 2017)

González, S. (2011). "Sistemas integrados de gestión, un reto para las pequeñas y medianas empresas", Escenarios, Vol. 9, No. 9, pp. 69-89

INEN. (2005). NTE INEN-ISO/IEC 17025:2005, Requisitos generales para la competencia de los laboratorios de ensayo y calibración (1ra ed). Ecuador: INEN.

ISO. (2015, a). ISO 9000:2015, Sistemas de gestión de la calidad Fundamentos y vocabulario (1ra ed). Ginebra - Suiza: ISO

ISO. (2015, b). ISO 14001:2015, Sistemas de gestión ambiental — Requisitos con orientación para su uso (1ra ed). Ginebra - Suiza: ISO 


\section{BIOGRAFÍAS}

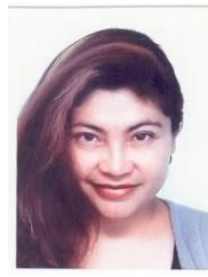

Mónica Susana Delgado. Graduada de Ingeniería Civil de la Escuela Politécnica Nacional, con un Master en Sistemas de Información Geográfica por parte de la Universidad de Salzburgo, una Maestría de Gestión Ambiental en UISEK, y Una Maestría en Administración Ambiental en proceso de reconocimiento. Desde el año 1997 fue asistente de investigación para el proyecto AARAM. A partir del año 1999 trabajó en el laboratorio de ingeniería ambiental de la facultad de ingeniería Civil y Ambiental de la EPN, ahora CICAM. De 2001 a 2015, trabajó como consultor independiente en Medio Ambiente y Gestión de Riesgos, e Hidrología. Especialista Geomático 3 en el Intituto Espacial Ecuatoriano, y luego director de investigación en INAMHI, Asesor de proyectos para funcionarios de la Secretaría de Gestión de Riesgos.

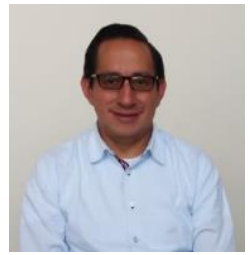

Marcelo F. Cabrera J. Nacido el 05 de marzo de 1986 en Quito-Ecuador, fue el mejor graduado de la Unidad Educativa "Jean Jacques Rousseau", promoción 2003-2004. Continuó sus estudios de pregrado en la Escuela Politécnica Nacional, obteniendo el título de Ingeniero Químico Aprobado Cum Laude. Finalmente obtuvo su título de posgrado en la Universidad Internacional SEK como Magíster en Gestión Ambiental. Ha trabajado en Incinerox, Chemeng y Escuela Politécnica Nacional donde hasta la actualidad labora como docente en la Facultad de Ingeniería Química y Agroindustria.

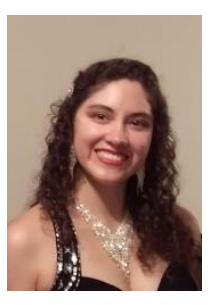

Gabriela Pérez. Nacida el 05 de octubre de 1987 en Quito- Ecuador, se graduó con honores en el Colegio Experimental 24 de Mayo. Es Ingeniería Química Cum Laude, realizó como proyecto de titulación el escalado del tamaño de pellet obtenido en la fermentación sumergida de sacarosa con Aspergillus niger, desde un reactor de 2 litros hasta uno de 14 litros, manteniendo constante el coeficiente Kla de transferencia de masa. Magister en Ingeniería Industrial y Productividad. Ganadora del concurso de méritos y oposición para Especialista en Técnicas de Análisis Químico. Todos sus estudios superiores y merecimientos los ha realizado en la Escuela Politécnica Nacional. 